\title{
A Novel Performance Measure for Picture Rate Conversion Methods
}

\author{
Adrienne Heinrich, Gerard de Haan, Claus Nico Cordes \\ Philips Research Laboratories, Eindhoven, The Netherlands
}

\begin{abstract}
Motion compensated interpolation (MCI) is crucial for motion portrayal improvement of modern displays, and film judder elimination. As MCI complexity grows, subjective optimization becomes cumbersome and elaborate. We present an objective metric that matches perception better than earlier measures and apply it to evaluate recent MCI algorithms.
\end{abstract}

\section{INTRODUCTION}

Modern television sets display video content at $50 \mathrm{~Hz}$ up to $120 \mathrm{~Hz}$. In order to prevent flicker and to improve the motion portrayal, movie material which is shot at $24 \mathrm{~Hz}$, $25 \mathrm{~Hz}$ or $30 \mathrm{~Hz}$ is up-converted to a higher frame rate (e.g. $100 \mathrm{~Hz}, 120 \mathrm{~Hz}$ ) by creating one or more images between two successive original images. Straightforward MCI algorithms simply repeat the images which causes motion judder and blur. More sophisticated algorithms use motion estimation (ME) and compensation [5] in order to improve the quality of the interpolated images.

Despite the improvement on smooth motion portrayal, motion compensated interpolation (MCI) often introduces artifacts due to incorrect motion vectors or sub-optimal interpolation methods [1]. These are often visible at the fore-background transitions of moving objects, i.e. in occlusion areas. As the complexity of these interpolation algorithms grows, the optimization task becomes increasingly time consuming when only subjective criteria are used. Yet a number of articles found in literature only use subjective observations as their evaluation measure (e.g. [6]).

In the past, performance measures have successfully been used for optimizing ME methods (e.g. [3]). Also for MCI, a representative metric may assist in speeding up the design phase and allow the evaluation and comparison of various methods. Previous studies proposed to accelerate original video sequences by skipping pictures [4], to reconstruct these by temporal upconversion - using the same factor as for the acceleration -, and to compare the original and the reconstructed pictures through the 'Mean Squared Error' (MSE). Yet, the drawback of this method is that the smallest integer acceleration (a factor of 2) already renders a sequence unrealistic. Furthermore, noninteger up-conversion factors cannot be tested at all. Our new proposed metric allows an evaluation of MCI on arbitrary sequences at their original speed. Additionally, it eliminates an important weakness of the MSE by taking into account that locally clustered errors are more disturbing than errors that are globally distributed over the interpolated image.

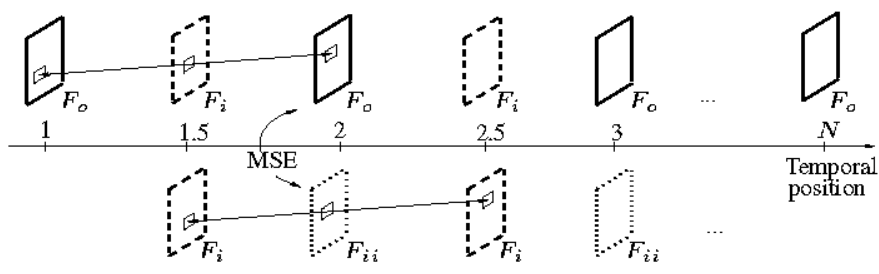

Fig. 1. MSE computation after double interpolation. The solid frames show the original input frames $F_{o}$ at the temporal positions $1,2, \ldots, N$, the dashed frames the first interpolation results $F_{i}$ at the temporal positions $1.5,2.5, \ldots$, $N-0.5$ and the dotted frames the double interpolated images $F_{i i}$.

\section{DESIGN OF A NEW PERFORMANCE MEASURE}

\section{A. Double interpolation}

In order to examine if MCI methods perform well, we propose a novel performance measure that allows a comparison between original and reconstructed images without altering the original speed of the test sequence. To this end, a twostep approach is suggested where a double interpolation is performed: the first interpolation takes place between original images and the second one on the interpolated result as illustrated in Fig. 1. Temporally up-converting a sequence of already interpolated images amplifies the errors which allows a good performance discrimination between two algorithms and returns an interpolated result at the position of original frames. The MSE can then be computed between double interpolated images and the original frames at the same temporal position $n$ using

$$
\operatorname{MSE}(n)=\frac{1}{f_{w} f_{h}} \sum_{x, y} \underbrace{\left(F_{o}(x, y, n)-F_{i i}(x, y, n)\right)^{2}}_{\mathrm{SE}},
$$

where $f_{w}$ and $f_{h}$ are the frame width and height respectively, $x$ and $y$ the pixel coordinates, $F_{o}$ and $F_{i i}$ the luminance values of the original input frames and double interpolated frames respectively.

\section{B. Capturing the most visible artifacts}

Especially occlusion areas pose a challenge for MCI. When the interpolation method cannot tackle the occlusion problem, severe artifacts are visible at the boundaries of moving objects [1]. Thus, the aim in the metric design is to emphasize these kind of artifacts which are indicated by large squared error (SE) values.

As the human eye is less sensitive to higher spatial frequencies, as shown by the contrast sensitivity function in [2], we focus 

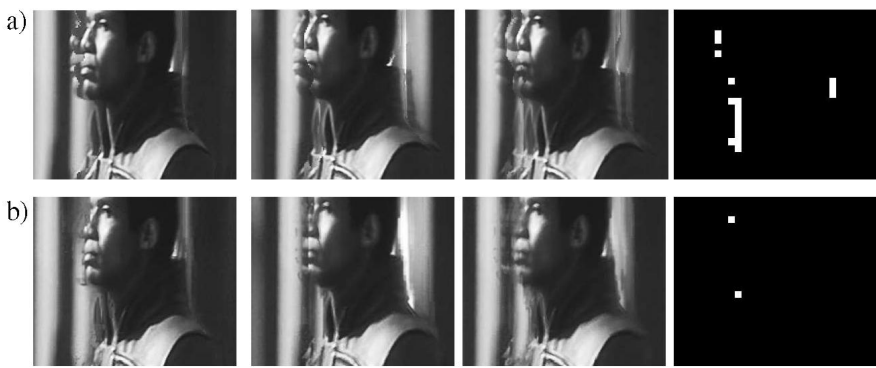

Fig. 2. Illustration of the performance measure: a) MCA and b) HR; from left to right respectively: two consecutive interpolated images $F_{i}$, double interpolated image $F_{i i}$ resulting from up-converting the first two images, the blocks $e_{b}$ above the error threshold $T_{e}$.

on the low frequent part of the image spectrum by comparing the DC error of image blocks on a block-by-block basis. The DC error $e_{b}$ for the block $b$ of $m_{b} \times n_{b}$ pixels with coordinates $(r, s, n)$ is computed as

$$
e_{b}(r, s, n)=\frac{1}{m_{b} n_{b}} \sum_{k=0}^{m_{b}} \sum_{l=0}^{n_{b}} \mathrm{SE}\left(r m_{b}+k, s n_{b}+l\right) .
$$

Since the disturbing artifacts in occlusion areas are exposed more by high magnitude errors and are hardly visible when the errors are small, only errors above a predefined threshold $T_{e}$ are taken into account. The 'Number of Significant Errors' is counted per frame and its mean, NSE, determined for all the double interpolated images as given by

$$
\mathrm{NSE}=\frac{1}{N_{i i}} \sum_{n=1}^{N_{i i}} \sum_{r, s} \frac{\operatorname{sgn}\left(e_{b}(r, s, n)-T_{e}\right)+1}{2},
$$

where $N_{i i}$ is the number of double interpolated images. If the error count NSE is very low the interpolation method is quantitatively regarded as good. A method introducing large boundary artifacts will generate a higher error count above the error threshold compared to a method performing well in occlusion regions. This can be observed by comparing the performance of two MCI methods 'Halo-reduced temporal interpolation' (HR) [1] and 'Motion-Compensated Averaging' (MCA) [5] on an example sequence of a walking man in Fig. 2. It is clearly visible that when the sophisticated HR method is employed, the less severe interpolation artifacts correlate well with the low NSE.

\section{RESULTS}

For the evaluation of the performance measure, twelve expert viewers compared and rated the metric results with preliminary subjective evaluations - in the form of blind sideby-side comparisons on a 42 inch LCD screen - of three MCI methods with several test sequences. The first method is MCA, the second one 'Dynamic Median filtering' (DM) [5] which was designed to improve the temporal up-conversion performance of stationary text and logos. The third MCI method is the sophisticated HR algorithm, which is capable of preventing image degradation in occlusion areas effectively.
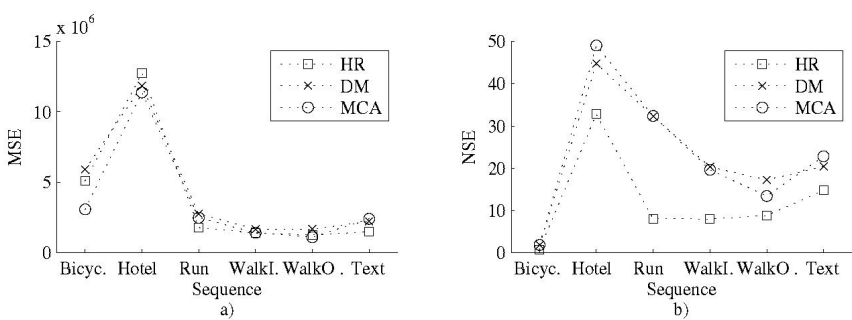

Fig. 3. a) MSE measure given by Eq. (1) and b) 'Number of Significant Errors' (NSE) given by Eq. (3) for several sequences and for the MCI methods HR, DM and MCA.

The test sequences comprise relatively simple content (Bicycle) and challenging content due to strong occlusion (Hotel, Run, WalkIn, WalkOut, Text), due to many fine details (Hotel), or due to subtitles with a moving background (Text). In Fig. 3, the NSE (see Eq. (3)) and the MSE (see Eq. (1)) between the double interpolated images and the original frames are depicted per sequence.

Indeed, the subjective evaluation corresponds significantly better with the NSE than with the mere MSE metric. This is particularly visible in sequences with challenging occlusion areas and fine details such as Hotel, where the MSE plot falsely indicates that HR is the worst performing method. Furthermore, the high MSE values of Bicycle lead to the misconception that it is a very challenging sequence. An additional benefit of the NSE is the clear distinction in performance of the different individual methods. Regarding the NSE, all MCI methods perform well on the simple Bicycle sequence, HR however clearly outperforms the other methods in the challenging occlusion sequences as also subjectively confirmed. In correspondence with the visual observation, the NSE reflects a better performance of DM than of MCA in stationary text (see Text) and fine detail (see Hotel).

\section{CONClusion}

A quantitative performance measure is beneficial for the design and optimization of MCI methods. In this paper, we have presented a performance measure which takes into account the most visible artifacts that frequently occur in occlusion regions. It evaluates $\mathrm{MCI}$ methods without increasing the original speed of the test sequence in contrast to the earlier performance measure. More thorough testing is needed to confirm the high correlation with the subjective assessment.

\section{REFERENCES}

[1] E. B. Bellers et al., "Solving occlusion in Frame-Rate up-Conversion", Digest of the ICCE, January 2007, pp. 1-2.

[2] P. G. J. Barten, Contrast Sensitivity of the Human Eye and its Effect on Image Quality, ISBN: 90-9012613-9, Knegsel, HV Press, 1999.

[3] G. de Haan et al., "True-Motion Estimation with 3-D Recursive Search Block Matching", IEEE Transactions on Circuits and Systems for Video Technology, Vol. 3, No. 5, October 1993, pp. 368-379.

[4] K. Hilman et al., "Using Motion-Compensated Frame-Rate Conversion for the Correction of 3:2 Pulldown Artifacts in Video Sequences", IEEE Trans. Circuits Syst. Video Technol., Vol. 10, No. 6, September 2000, pp. 869-877.

[5] O. A. Ojo et al., "Robust motion-compensated video up-conversion", IEEE Tr. on CE, Vol. 43, No. 4, November 1997, pp. 1045-1055.

[6] H. Sonehara et al., "Reduction of motion judder on video images converted from film", SMPTE J., Vol. 106, 1997, pp. 535-540. 